\title{
ANOMALOUS BEHAVIOR OF THE HALL EFFECT IN III-V HETEROSTRUCTURES
}

\author{
Z. DzIuba ${ }^{a *}$, M. GóRsKA ${ }^{a}$, J. MARCzeWSKI $^{b}$, T. PrzeseaWski ${ }^{b}$ \\ AND K. REGIŃSKI ${ }^{b}$ \\ anstitute of Physics, Polish Academy of Sciences \\ Al. Lotników 32/46, 02-668 Warsaw, Poland \\ ${ }^{b}$ Institute of Electron Technology, Al. Lotników 32/46, 02-668 Warsaw, Poland
}

(Received August 31, 1999; revised version December 2, 1999)

The Hall effect and magnetoresistance were measured in the InAs/GaAs heterostructure at temperatures from $300 \mathrm{~K}$ down to $3 \mathrm{~K}$, in a magnetic field range from 0.01 to $1.5 \mathrm{~T}$. The anomalous magnetic field dependence of the Hall coefficient in the InAs/GaAs heterostructure in magnetic fields below $0.1 \mathrm{~T}$ was explained as due to an extraordinary Hall effect caused by skew scattering on dislocations.

PACS numbers: $61.72 . \mathrm{Lk}, 72.80 . \mathrm{Ey}$

\section{Introduction}

Thin, undoped heterostructures are very interesting from the point of view of their transport properties. In such samples the contribution of the conduction band or valence band carriers to the conductivity can be small and effects associated with the interface can become dominant. In our previous paper we showed that in a thin, undoped $\operatorname{In}_{0.53} \mathrm{Ga}_{0.47} \mathrm{As} / \mathrm{InP}$ heterostructure nearly all the electrical conduction takes place in the impurity band at the interface [1]. The electrical conduction in the impurity band was about $95 \%$ of the total electrical conduction. It was suggested there that the impurity band originated from unintentionally introduced impurities and interfacial dislocations.

The effect of dislocations on the electrical conduction in GaAs-based compounds has been widely investigated [2-12]. However, skew scattering has not been taken into account. It is well known that at the interface there is a high density of dislocations and we can expect a significant influence of dislocation on the electrical conduction in the layer in which the interface conduction is dominant. In the analysis of the electrical conduction, dislocations have been considered as

*corresponding author; e-mail: dziuba@ifpan.edu.pl 
scattering centers. The magnetic properties of structure defects, i.e. the ferromagnetic $[13,14]$, antiferromagnetic [15], and paramagnetic phases [16-18] associated with dislocations have been experimentally and theoretically investigated. The interaction of electric carriers with magnetic moments leads to the extraordinary Hall effect widely investigated in materials with magnetic atoms [19-29]. Then, in highly mismatched heterostructures we can expect the extraordinary Hall phenomena, as in case of crystals with magnetic atoms.

\section{Preparation of the sample and measurements}

Semi-insulating GaAs substrates were used for MBE grown $0.67 \mu \mathrm{m}$ GaAs undoped layer and $4 \mu \mathrm{m}$ undoped InAs layer. Our investigation shows that in InAs layer, mismatch dislocations penetrate about $2 \mu \mathrm{m}$ of the InAs epilayer. Then, we can expect the electrical properties of the part of the InAs layer close to interface InAs/GaAs different from the properties of the other part of the InAs layer.

The Hall coefficient and magnetoresistance in the InAs/GaAs heterostructure at temperatures from $10 \mathrm{~K}$ to $292 \mathrm{~K}$ were measured in magnetic fields up to $0.6 \mathrm{~T}$ using the Van der Pauw method. The results of the measurements in form of the sheet Hall coefficient and of the magnetoresistance are shown in Fig. 1.
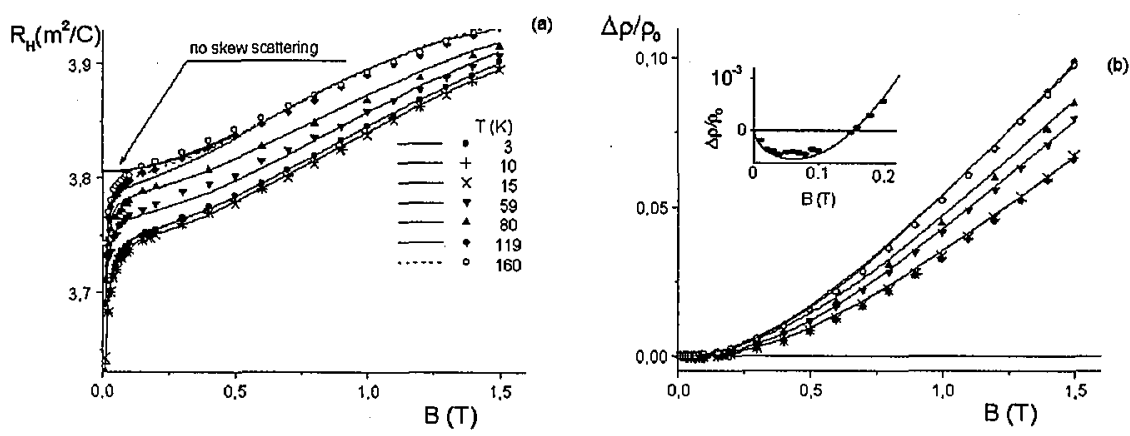

Fig. 1. Magnetic field dependence of the sheet Hall coefficient (a) and magnetoresistance (b) in the InAs/GaAs heterostructure. Experimental data - points, theoretical curves - thin lines, results of calculation without skew scattering for $T=160 \mathrm{~K}-$ thick line. Inset in Fig. 1b - magnetoresistance at $T=3 \mathrm{~K}$.

In Fig. 1a we can see a very unusual magnetic field dependence of the sheet Hall coefficient $\left(R_{\mathrm{H}}\right)$. Below $0.1 \mathrm{~T}$ we observe a sharp increase in $R_{\mathrm{H}}$ at all investigated temperatures. The non-linear magnetic field dependence of the Hall coefficient in low magnetic fields, shown in Fig. 1a, was not previously observed, except in a multilayer AlGaAs/GaAs structure [30]. Recently, we have observed similar effect in an AlGaN/GaN heterostructure [31], and even in a well-matched $\mathrm{In}_{0.53} \mathrm{Ga}_{0.47} \mathrm{As} / \mathrm{InP}$ heterostructure [32].

In Fig. 1b we can see a small negative magnetoresistance in low magnetic field at temperatures below $59 \mathrm{~K}$. Negative magnetoresistance has been widely observed in many semiconductors and magnetic materials $[1,25,26]$. 


\section{Model of the electrical conduction}

The anomalous magnetic field dependence of the Hall coefficient in magnetic fields below $0.1 \mathrm{~T}$, shown in Fig. 1a, cannot be explained using only the standard transport theory. On the other hand, from all possible quantum phenomena, only the skew scattering seems to be a most reasonable explanation of the anomalous Hall coefficient in the InAs/GaAs heterostructure. We assume that the magnetic field dependence of the Hall coefficient below $0.1 \mathrm{~T}$ can be explained as resulting from interaction of carriers with magnetic moments of interfacial dislocations. The interaction of the electrical carriers with the magnetic moments leads to the widely investigated extraordinary Hall effect. In such case, the total Hall coefficient contains two terms and has the form

$$
R=R_{\text {ord }}+R_{\text {extr }} \frac{M(B)}{B},
$$

where $R, R_{\text {ord }}$, and $R_{\text {extr }}$ are the total, ordinary, and the extraordinary Hall coefficient, respectively, $B$ is the magnetic induction, and $M(B)$ is the magnetization. The extraordinary Hall coefficient, $R_{\text {extr }}$, is independent of the magnetic field and of the sign of the ordinary Hall coefficient. It can be positive or negative in the same magnetic material $[19,20]$.

In the analysis of the electrical conduction in multilayer structures, a mixed conduction should be taken into account. Magnetic field dependence of the Hall coefficient above $0.1 \mathrm{~T}$ and positive magnetoresistance are due to a mixed type conduction in the investigated InAs/GaAs heterostructure. Small, negative magnetoresistance was explained as a localization effect. Localization effect and extraordinary Hall voltage were considered as quantum corrections to the classically described electrical conduction.

Our preliminary analysis of the electrical conduction in the InAs/GaAs heterostructure shows that three groups of carriers describe magnetic field dependence of the Hall coefficient and magnetoresistance. We assumed that quantum effects modify the electrical conduction of all three groups of carriers. In our model, the mixed electrical conduction was described using conductivity tensor components modified by quantum effects in the form

$$
\begin{aligned}
\sigma_{x x} & =\sum_{i=1}^{3} \frac{\sigma_{i}(B)}{1+\left[\mu_{i}(B) B+\mu_{\mathrm{S}} P_{\mathrm{S}}\right]^{2}}, \\
\sigma_{x y} & =\sum_{i=1}^{3} \frac{\sigma_{i}(B)\left[\mu_{i}(B) B+\mu_{\mathrm{S}} P_{\mathrm{S}}\right]}{1+\left[\mu_{i}(B) B+\mu_{\mathrm{S}} P_{\mathrm{S}}\right]},
\end{aligned}
$$

where $\sigma_{i}(B)=e n_{i} \mu_{i}(B)$ is the sheet conduction, $n_{i}$ is the sheet concentration, $\mu_{i}(B)$ is the mobility [1], $e$ is the electric charge, $P_{\mathrm{S}}=\mu_{0} M_{\mathrm{S}}$ is the magnetic polarization, $M_{\mathrm{S}}$ is the saturation magnetization, assumed to be constant, $\mu_{0}$ is the permeability of free space, and $\mu_{\mathrm{S}}$ is the coefficient describing effect of the interaction of carriers with magnetic moment. In Eq. (2) $\mu_{\mathrm{S}} P_{\mathrm{S}}$ is the Hall angle associated with the extraordinary Hall effect.

The Hall coefficient, $R(B)$, and magnetoresistance, $\Delta \rho / \rho_{0} \rho(B) / \rho\left(B_{0}\right)-1$, were calculated by using conductivity tensor components shown in Eq. (2). The 

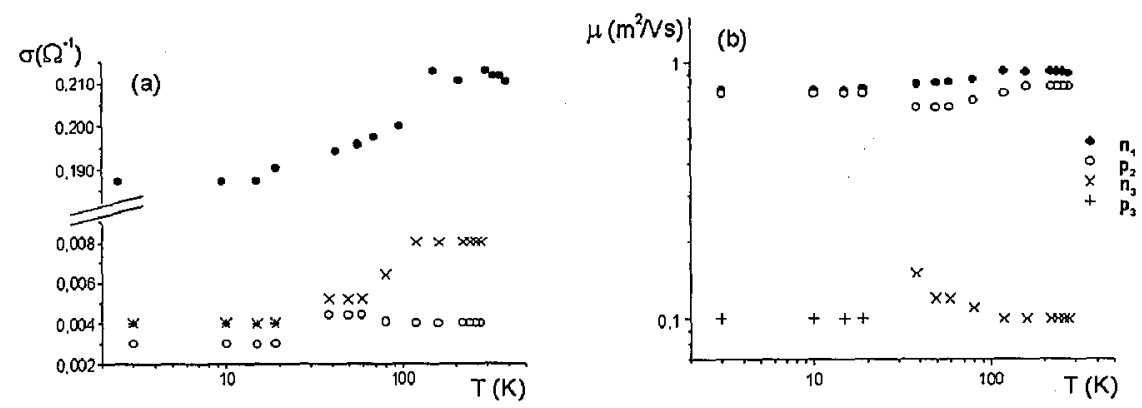

Fig. 2. The temperature dependence of the sheet conductivities (a) and mobilities (b) estimated for the InAs/GaAs heterostructure. $n_{1}$ - first group of carriers (electron-like), $p_{2}$ - second group of carriers (hole-like), $n_{3}$ and $p_{3}$ - third group of carriers (not analyzed $n$-type and $p$-type contributions).

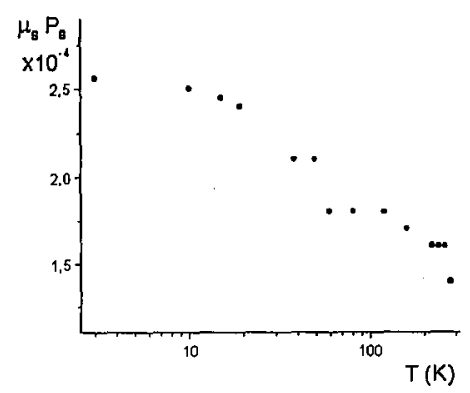

Fig. 3. The temperature dependence of the extraordinary Hall angle, $\mu_{\mathrm{S}} P_{\mathrm{S}}$, for In As/GaAs heterostructure.

Hall coefficient calculated for case of one group of carriers contains ordinary, $R_{\text {ord }}=\mu / \sigma$, and extraordinary, $R_{\text {extr }}=\mu_{\mathrm{S}} \mu_{0} / \sigma$, components.

The calculated Hall coefficient and magnetoresistance are shown in Fig. 1 by lines. The inset in Fig. 1b shows a very small, however non-negligible, negative magnetoresistance. To show the effect of dislocations the Hall coefficient was calculated for $M_{\mathrm{S}}=0$ and the result for temperature $T=275 \mathrm{~K}$ is shown in Fig. 1a by the thick solid line. The conductivities and mobilities of three groups of carriers used as the fitting parameters to the experimental data are shown in Fig. 2, and the Hall angle, $\mu_{\mathrm{S}} P_{\mathrm{S}}$, as a function of temperature is shown in Fig. 3.

\section{Comments on the electrical conduction in InAs/GaAs}

The conductivities and mobilities of all three groups of carriers are shown in Fig. 2. Two groups of carriers have mobilities of about $0.8 \mathrm{~m}^{2} /(\mathrm{V} \mathrm{s})$ and another group of carriers has a mobility of about $0.1 \mathrm{~m}^{2} /(\mathrm{V} \mathrm{s})$. According to the analysis of the electrical conduction in the $\mathrm{HgTe}[33,34], \mathrm{GaN} / \mathrm{AlGaN}$ [35], and $\operatorname{In}_{0.53} \mathrm{Ga}_{0.47} \mathrm{As} / \mathrm{InP}$ [1] it is easy to show that the electrons in InAs at temperature below $100 \mathrm{~K}$ with the mobility about $0.8 \mathrm{~m}^{2} /(\mathrm{V} \mathrm{s})$ cannot be in the conduction band. Then, electron-like $\left(n_{1}\right)$ and hole-like $\left(p_{2}\right)$ carriers with mobilities about 
$0.75 \mathrm{~m}^{2} /(\mathrm{V} \mathrm{s})$ were ascribed to the impurity band. The nature of carriers with mobility of $0.1 \mathrm{~m}^{2} /(\mathrm{V} \mathrm{s})$ has not been analyzed in this paper.

The density of the impurity band electrons, $n$, was estimated by using the sheet conduction $\sigma_{n_{1}}=0.2 \Omega^{-1}$, the mobility $\mu_{n_{1}}=0.8 \mathrm{~m}^{2} /(\mathrm{V} \mathrm{s})$, and the thickness of the InAs layer with dislocations, $d=2 \mu \mathrm{m}$. In our case $n=1.5 \times 10^{24} \mathrm{~m}^{-3}$. Usually, in an undoped InAs/GaAs heterostructure the concentration of carriers would not be so high. Therefore, it can be assumed that the impurity band originates from dislocations. In general, in the impurity band, the mobility of carriers is proportional to the concentration of structure defects producing impurity band states. From comparison of the mobility of impurity band carriers in $\mathrm{In}_{0.53} \mathrm{Ga}_{0.47} \mathrm{As} / \mathrm{InP}\left(0.3 \mathrm{~m}^{2} /(\mathrm{V} \mathrm{s})\right)[1]$ and InAs/GaAs $\left(0.8 \mathrm{~m}^{2} /(\mathrm{V} \mathrm{s})\right)$ heterostructures it can be concluded that the concentration of structure defects which form the impurity band is higher in the InAs/GaAs heterostructure. This is consistent with our assumption of a significant contribution of dislocations to the density of states in the impurity band in both heterostructures.

\section{Conclusions}

Our results indicate that the anomalous behavior of the Hall effect in In As/GaAs can be interpreted as the extraordinary Hall effect associated not with magnetic ions but with structure defects. Carriers in the impurity band dominate the electrical conduction in the investigated InAs/GaAs heterostructure. The impurity band is formed mainly by quantum states associated with dislocations. In the impurity band the $n$-like conduction gives $95 \%$ and the $p$-like conduction about $2 \%$ of total conduction. Both components have nearly the same mobilities of about $0.75 \mathrm{~m}^{2} /(\mathrm{V} \mathrm{s})$.

\section{Acknowledgments}

The authors thank Professor T. Figielski and Dr. T. Wosiński for useful discussions. This work was partially supported by the Committee for Scientific Research under grant PBZ 28. 11/P7.

\section{References}

[1] Z. Dziuba, T. Przesławski, K. Dybko, M. Górska, J. Marczewski, K. Regiński, J. Appl. Phys. 85, 6619 (1999).

[2] A.H. Eltoukhy, J.E. Greene, J. Appl. Phys. 50, 6396 (1979).

[3] Q. Zhu, K. Hiramatsu, N. Sawaki, I. Akasaki, Jap. J. Appl. Phys. 27, 1674 (1988).

[4] V.W.L. Chin, T.L. Tansley, Solid State Electron. 34, 1055 (1991).

[5] P. Hiesinger, T. Schweizer, K. Kohler, P. Ganser, W. Rothemund, W. Jantz, J. Appl. Phys. 72, 2941 (1992).

[6] M.J. Ekenstedt, P. Songpongs, T.G. Andersson, Appl. Phys. Lett. 61, 789 (1992).

[7] Y. Uchida, H. Kakibayashi, S. Goto, J. Appl. Phys. 74, 6720 (1993).

[8] S.Z. Chang, T.C. Chang, J.L. Shen, S.C. Lee, Y.F. Chen, J. Appl. Phys. 74, 6912 (1993).

[9] C. Besikci, Y.H. Choi, G. Labeyrie, E. Bigan, M. Razeghi, I.B. Cohen, J. Carsello, V.P. Dravid, J. Appl. Phys. 76, 5820 (1994). 
[10] Z. Bougrioua, J.L. Farvacque, D. Ferre, J. Appl. Phys. 79, 1536, 1546 (1996).

[11] H. Yamaguchi, Y. Hirayama, Jap. J. Appl. Phys. 37, 1599 (1998).

[12] V. Gopal, E.P. Kram, T.P. Chin, J.M. Woodall, Appl. Phys. Lett. 72, 2319 (1998).

[13] A.M. Kosevich, V.A. Shklovsky, Zh. Exp. Teor. Fiz. 55, 1131 (1968).

[14] G.R. McClurg, C.F. Squire, J. Chem. Phys. 51, 631 (1969).

[15] T. Nakamura, K. Kawamura, J. Magn. Magn. Mater. 31-34, 1483 (1983).

[16] T. Wosiński, T. Figielski, Phys. Status Solidi B 83, 93 (1977).

[17] D. Neubert, K. Hoffmann, H. Teichmann, R. Schlief, Solid State Electron. 21, 1445 (1978).

[18] L.S. Vlasenko, Physica B \&C 116B+C, 281 (1983).

[19] P. Karplus, J.M. Luttinger, Phys. Rev. 95, 1154 (1954).

[20] J.M. Luttinger, Phys. Rev. 112, 739 (1958).

[21] A. Fert, P.M. Levy, Phys. Rev. B 36, 1907 (1987).

[22] A.M. Sandauer, Phys. Status Solidi A 111, k219 (1989).

[23] H. Sato, H. Henmi, Y. Kobayashi, Y. Aoki, H. Yamamoto, T. Shinjo, V. Sechovsky, J. Appl. Phys. 76, 6919 (1994).

[24] P. Łazarczyk, T. Story, A. Jędrzejczak, R.R. Gałązka, W. Mac, M. Herbich, A. Stachow-Wójcik, J. Magn. Magn. Mater. 176, 233 (1997).

[25] A. Oiva, A. Endo, S. Katsumoto, Y. Iye, H. Ohno, H. Munekata, Phys. Rev. B 59, 5826 (1999).

[26] F. Matsukura, H. Ohno, A. Shen, Y. Sugawara, Phys. Rev. B 57, R2037 (1998-II).

[27] H. Ohno, A. Shen, F. Matsukura, A. Oiwa, A. Endo, S. Katsumoto, Y. Iye, Appl. Phys. Lett. 69, 363 (1996).

[28] S. Zhang, Phys. Rev. B 51, 3632 (1995).

[29] N. Ryzhanova, A. Vedaye, A. Crepieux, C. Lacroix, Phys. Rev. B 58, R2933 (1998).

[30] W.A. Beck, R.A. Wilson, A.C. Goldberg, J. Cryst. Growth 81, 136 (1987).

[31] J. Antoszewski, A. Bobiński, Z. Dziuba, unpublished data.

[32] T. Przesławski, K. Regiński, unpublished data.

[33] Z. Dziuba, K. Szlenk, J. Phys. Chem. Solids 45, 97 (1984).

[34] Z. Dziuba, Phys. Status Solidi B 117, 531 (1983); Phys. Status Solidi B 118, 319 (1983).

[35] Z. Dziuba, J. Antoszewski, J.M. Dell, L. Faraone, P. Kozodoy, S. Keller, S.P. DenBaars, U.K. Mishra, J. Appl. Phys. 82, 15 (1997). 